\title{
Adaptive Extended Kalman Filter in SINS/GPS Tightly Coupled Navigation System
}

\author{
Zang Zhongyuan ${ }^{1,}$, Miao Lingjuan ${ }^{2, b}$ and Guo Yanbing ${ }^{3, c}$ \\ ${ }^{1,2,3}$ School of Automation, Beijing Institute of Technology, Beijing, 100081, China \\ alibaren@163.com, ${ }^{\text {b }}$ miaolingjuan@bit.edu.cn, 'cggb1096@126.com
}

Keywords: SINS/GPS tightly integrated navigation system; GDOP; state noise; measurement noise; adaptive EKF

\begin{abstract}
In the practical application, the SINS/GPS tightly integrated navigation system is faced with the situation that state error model is inaccurate or noise statistical characteristics are inconsistent with the reality. However, traditional Extended Kalman Filter (EKF) method can not effectively solve it, causing large filter errors in this system. This paper presents a method of GDOP estimation and adaptive Extended Kalman Filter, which combines satellite positions of ephemeris with the output of corrected SINS position instead of GPS positioning results to calculate GDOP; On this basis, using the GDOP value and innovation realizes the online real-time estimation of measurement noise variance matrix $R_{k}$ while taking advantage of innovation realizes the online estimation of state noise variance matrix $Q_{k}$ to achieve the adaptive effect and improve navigation accuracy in tightly integrated navigation system.
\end{abstract}

\section{Introduction}

Strap-down Inertial Navigation System (SINS) does not depend on any external information, autonomy is strong and data update speed is fast, but the error of SINS will diverge over time, so SINS is not suitable for long time navigation; Global Positioning System (GPS) can work with high precision and long term, its error does not diverge over time, but there are also some shortcomings such as multipath effects and easily-affected radio interference, resulting in low stability of navigation. Therefore, GPS can't perform well as a standalone system. In the high accuracy navigation field, GPS and SINS are mostly integrated together to overcome their own shortcomings, which makes the navigation performance better than the performance of two separate systems. SINS/GPS tightly integrated navigation system uses more raw data from GPS receiver pseudo-ranges and pseudo-range rates and has obvious advantages. Therefore, this paper focuses on the study of SINS/GPS tightly coupled system in order to achieve better navigation performance.

In practical applications, due to the error of inertial device itself and inaccurate external environment information, there is a system error model inaccuracy and noise uncertainty in the SINS/GPS nonlinear systems. As for the uncertainty of system noise and statistical properties of measurement noise, people proposed various adaptive filtering algorithms. Literature [2-4] proposed a method of real-time estimating the system noise and measurement noise based on the innovation. The method does not increase the system dimensionality, only needs limited innovation to memory, and the calculation is simple and reliable, but cannot effectively solve the question that the system noise and measurement noise are inaccurate at the same time. This paper presents an adaptive filtering method for online estimation of the measurement noise covariance matrix $\boldsymbol{R}_{\boldsymbol{k}}$ and the system noise covariance matrix $\boldsymbol{Q}_{\boldsymbol{k}}$ based on estimated GDOP value and innovation in EKF, and then applying this method to the SINS/GPS tightly coupled navigation system and verifying the validity of the adaptive algorithm.

\section{SINS/GPS tightly integrated system model}

State equation of integrated system is as follows ${ }^{[5]}$ : 


$$
\dot{\boldsymbol{X}}=\left[\begin{array}{c}
\dot{\boldsymbol{X}}_{\text {SINS }} \\
\dot{\boldsymbol{X}}_{G P S}
\end{array}\right]=\left[\begin{array}{cc}
\boldsymbol{F}_{S I N S} & \boldsymbol{0} \\
\boldsymbol{0} & \boldsymbol{F}_{G P S}
\end{array}\right]\left[\begin{array}{l}
\boldsymbol{X}_{\text {SINS }} \\
\boldsymbol{X}_{G P S}
\end{array}\right]+\left[\begin{array}{cc}
\boldsymbol{G}_{\text {SINS }} & \boldsymbol{0} \\
\boldsymbol{0} & \boldsymbol{G}_{G P S}
\end{array}\right]\left[\begin{array}{l}
\boldsymbol{W}_{S I N S} \\
\boldsymbol{W}_{G P S}
\end{array}\right]=\boldsymbol{F} \boldsymbol{X}+\boldsymbol{G W}
$$

The error state variable $\boldsymbol{X}$ represents the position, velocity, and attitude errors of a vehicle, the inertial sensor bias errors which consist of the accelerometer bias errors and the gyro bias errors, the clock error and clock drift error from GPS, while $\boldsymbol{W}$ is the white noise of the inertial sensor errors, GPS clock error and clock drift error with zero mean. The information of the system matrix $\boldsymbol{F}_{\text {SINS }}, \boldsymbol{F}_{\text {GPS }}$, $\boldsymbol{G}_{\text {SINS }}$ and $\boldsymbol{G}_{\text {GPS }}$ is referred in [5].

The measurement model of the INS/GPS tightly coupled system uses the pseudo-range and pseudo-range rate information of the INS and the GPS:

$$
\boldsymbol{Z}=\left[\begin{array}{c}
\delta \boldsymbol{\rho} \\
\delta \dot{\boldsymbol{\rho}}
\end{array}\right]=\left[\begin{array}{c}
\boldsymbol{\rho}_{\text {sins }}-\boldsymbol{\rho}_{G} \\
\dot{\boldsymbol{\rho}}_{\text {sins }}-\dot{\boldsymbol{\rho}}_{G}
\end{array}\right]=\left[\begin{array}{c}
\boldsymbol{h}_{\boldsymbol{\rho}}(\boldsymbol{X}) \\
\boldsymbol{h}_{\dot{\boldsymbol{\rho}}}(\boldsymbol{X})
\end{array}\right]+\left[\begin{array}{c}
\boldsymbol{V}_{\boldsymbol{\rho}} \\
\boldsymbol{V}_{\dot{\boldsymbol{\rho}}}
\end{array}\right]=\left[\begin{array}{c}
\boldsymbol{H}_{\boldsymbol{\rho}} \\
\boldsymbol{H}_{\dot{\boldsymbol{\rho}}}
\end{array}\right] \boldsymbol{X}+\left[\begin{array}{c}
\boldsymbol{V}_{\boldsymbol{\rho}} \\
\boldsymbol{V}_{\dot{\boldsymbol{\rho}}}
\end{array}\right]=\boldsymbol{H} \boldsymbol{X}+\boldsymbol{V}
$$

Here, $\boldsymbol{V}$ is the white Gaussian noise with zero mean. The information of the system matrix $\boldsymbol{H}$ and $\boldsymbol{h}$ is referred in [5].

\section{GDOP estimation method of SINS/GPS tightly integrated system}

In SINS/GPS tightly integrated navigation system, the previous GDOP value was usually calculated by positioning results of the GPS receiver. However, in practical applications, satellite selection process will be repeated due to the change of visible satellites, which increases the computational time and impacting real-time demand of GDOP value. To resolve this problem, this paper puts forward a method that combines the revised position of SINS with the satellites position to compute the approximate value of GDOP, so as to complete the selection of satellites.

Refer to the methods of solving GDOP in the section 2.1 of Literature [5] and section 5.4 of Literature [6], the revised position of SINS is used to transform the following pseudo-range observation equation:

$$
\boldsymbol{\rho}-\boldsymbol{r}_{0}=\boldsymbol{A} \boldsymbol{X}+\boldsymbol{V} \quad \text { TO } \boldsymbol{\rho}-\boldsymbol{r}_{I}=\boldsymbol{A}_{I} \boldsymbol{X}+\boldsymbol{V}
$$

Here, $\boldsymbol{r}_{0}$ is the predicted value of pseudo-range vector; $\boldsymbol{\rho}$ and $\boldsymbol{r}_{I}$ are pseudo-range vectors which are respectively obtained from GPS and the revised position of SINS; the pseudo-range of satellite $j$ is determined by the adjusted SINS position: $r_{I}^{j}=\sqrt{\left(x_{s}^{j}-x_{I}\right)^{2}+\left(y_{s}^{j}-y_{I}\right)^{2}+\left(z_{s}^{j}-z_{I}\right)^{2}}, e_{I, \xi}^{j}=\left(\xi_{I}-\xi_{s}^{j}\right) / r_{I}^{j}$ (Here $\xi=x, y, z ; \xi_{I}$ indicates the adjusted SINS position in ECEF Coordinate System).The corresponding weight coefficient matrix is as follows: $\quad \boldsymbol{G}_{I}=\left(\boldsymbol{A}_{I}^{T} \boldsymbol{A}_{I}\right)^{-1}=\left\{g_{I, i j}\right\},(i, j=1,2,3,4)$.

Define geometric dilution of precision solved by the adjusted SINS position as $G D O P_{I}$, we can obtain:

$$
G D O P_{I}=\sqrt{g_{I, 11}+g_{I, 22}+g_{I, 33}+g_{I, 44}}
$$

In this paper, the number of visible satellites is four. In the integrated navigation system, the adjusted SINS position errors are small, thus we can use them to compute GDOP, and then complete the choice of satellites. The method makes full use of the known quantity of integrated navigation system, thus shortening the time of choosing satellites significantly.

\section{The online estimation of measurement noise covariance matrix $\boldsymbol{R}_{k}$ and system noise covariance matrix $Q_{k}$}

In the design of EKF filter, we usually assume that the system noise and measurement noise are known. However, it is difficult to establish the model of system noise and measurement noise precisely, which means it is impossible to accurately determine the variance matrix $\boldsymbol{Q}_{\boldsymbol{k}}$ and $\boldsymbol{R}_{\boldsymbol{k}}{ }^{[7][8]}$ in advance. Therefore we need to estimate $\boldsymbol{Q}_{\boldsymbol{k}}$ and $\boldsymbol{R}_{\boldsymbol{k}}$ online to improve the performance of SINS/GPS tightly integrated navigation system.

Online Estimation of the Measurement Noise Variance Matrix $\boldsymbol{R}_{k}$ 
Actually, GPS system will be influenced by many factors, such as various satellites, multipath effect, the blocked signals and internal measurement noise, which will change the measurement errors in the SINS/GPS tightly coupled navigation system, so we need to estimate the value of $\boldsymbol{R}_{\boldsymbol{k}}$ online. Actually, the definition of GDOP includes the error information of location solution. Therefore, this article presents a solution which uses the value of $\mathrm{GDOP}_{I}$ in formula (3) and estimation error covariance matrix $\hat{\boldsymbol{P}}_{\Delta \overline{\boldsymbol{X}}_{k}}{ }^{[9]}$ to amend the measurement noise variance matrix $\boldsymbol{R}_{\boldsymbol{k}}$ online.

Measurement error $\Delta \delta \boldsymbol{\rho}$, position error $\Delta \delta x, \Delta \delta y, \Delta \delta z$ and clock correction $\Delta \delta t_{u}$ have a relationship as follows: $\quad\left[\begin{array}{llll}\Delta \delta x & \Delta \delta y & \Delta \delta z & \Delta \delta t_{u}\end{array}\right]^{T}=\left(\boldsymbol{A}^{T} \boldsymbol{A}\right)^{-1} \boldsymbol{A}^{T} \Delta \delta \boldsymbol{\rho}$

We find that the left hand side of equation (4) could be replaced by some terms in $\Delta \overline{\boldsymbol{X}}_{k}$, which are related to position and clock correction. Because the value of $\mathrm{GDOP}_{I}$ could be achieved after choosing the satellites, so we could calculate the variance matrix $\sigma^{2}$ of $\Delta \delta \boldsymbol{\rho}$ at the right hand side of equation (4) and use the variance as an estimation of the measurement noise variance matrix $\boldsymbol{R}_{\boldsymbol{k}}$.

Because of

$$
\begin{aligned}
& E\left(\left[\Delta \delta x \Delta \delta y \Delta \delta z \Delta \delta t_{u}\right]^{T}\left[\Delta \delta x \Delta \delta y \Delta \delta z \Delta \delta t_{u}\right]\right) \\
& =\left(\boldsymbol{A}^{T} \boldsymbol{A}\right)^{-1} E\left(\Delta \delta \boldsymbol{\rho}(\Delta \delta \boldsymbol{\rho})^{T}\right)=\sigma^{2} \boldsymbol{G}_{I}
\end{aligned}
$$

So we have:

$$
\sigma^{2} \operatorname{tr}\left(\boldsymbol{G}_{I}\right)=\hat{\boldsymbol{P}}_{\Delta \bar{X}_{k}}(\delta x)+\hat{\boldsymbol{P}}_{\Delta \bar{x}_{k}}(\delta y)+\hat{\boldsymbol{P}}_{\Delta \bar{x}_{k}}(\delta z)+\hat{\boldsymbol{P}}_{\Delta \bar{x}_{k}}\left(\delta t_{r u}\right)
$$

Here, $\hat{\boldsymbol{P}}_{\Delta \bar{x}_{k}}(i)$ ( $i=\delta x, \delta y, \delta z, \delta t_{r u}$ ), which is matched to diagonal elements of $\hat{\boldsymbol{P}}_{\Delta \bar{x}_{k}}$ separately, indicating the estimation error variance of position error and clock error.

Furthermore, through formula (3) we could get:

$$
\sigma^{2}=\frac{\hat{\boldsymbol{P}}_{\Delta \bar{X}_{k}}(\delta x)+\hat{\boldsymbol{P}}_{\Delta \bar{X}_{k}}(\delta y)+\hat{\boldsymbol{P}}_{\Delta \bar{X}_{k}}(\delta z)+\hat{\boldsymbol{P}}_{\Delta \bar{X}_{k}}\left(\delta t_{r u}\right)}{G D O P_{I}^{2}}
$$

The observation error variance of pseudo-range rate, the estimation variance of velocity error and the estimation variance of clock error rate also have the same relationship.

Since the output of SINS includes 3D position and velocity in Geographic Coordinate System, we must transform $\boldsymbol{G}_{I}$ from ECEF Coordinate System to Geographic Coordinate System before estimating $\boldsymbol{R}_{\boldsymbol{k}}$, supposed as $\boldsymbol{G}_{I, \lambda L h}$ and $\boldsymbol{G}_{I, E N U}$, corresponding to $G D O P_{I, \lambda L h}$ and $G D O P_{I, E N U}$ respectively. Hence, the diagonal elements of corresponding measurement noise variance matrix could be expressed as:

$$
\begin{aligned}
& \boldsymbol{R}_{k}(\boldsymbol{\rho})=\frac{\hat{\boldsymbol{P}}_{\Delta \overline{\boldsymbol{X}}_{k}}(\delta x)+\hat{\boldsymbol{P}}_{\Delta \bar{x}_{k}}(\delta y)+\hat{\boldsymbol{P}}_{\Delta \overline{\boldsymbol{X}}_{k}}(\delta \mathrm{z})+\hat{\boldsymbol{P}}_{\Delta \overline{\boldsymbol{X}}_{k}}\left(\delta t_{r u}\right)}{G D O P_{I, \lambda L h}^{2}}=\frac{\hat{\boldsymbol{P}}_{\Delta \overline{\boldsymbol{X}}_{k}}(\delta x)+\hat{\boldsymbol{P}}_{\Delta \bar{x}_{k}}(\delta y)+\hat{\boldsymbol{P}}_{\Delta \bar{X}_{k}}(\delta z)+\hat{\boldsymbol{P}}_{\Delta \bar{X}_{k}}\left(\delta t_{r u}\right)}{\operatorname{tr}\left(\boldsymbol{G}_{I, \lambda L h}\right)} \\
& \boldsymbol{R}_{k}(\dot{\boldsymbol{\rho}})=\frac{\hat{\boldsymbol{P}}_{\Delta \overline{\mathbf{X}}_{k}}\left(\delta V_{E}\right)+\hat{\boldsymbol{P}}_{\Delta \bar{X}_{k}}\left(\delta V_{N}\right)+\hat{\boldsymbol{P}}_{\Delta \overline{\mathbf{X}}_{k}}\left(\delta V_{U}\right)+\hat{\boldsymbol{P}}_{\Delta \bar{X}_{k}}\left(\delta t_{r u}\right)}{G D O P_{I, E N U}^{2}}=\frac{\hat{\boldsymbol{P}}_{\Delta \overline{\mathbf{X}}_{k}}\left(\delta V_{E}\right)+\hat{\boldsymbol{P}}_{\Delta \overline{\mathbf{X}}_{k}}\left(\delta V_{N}\right)+\hat{\boldsymbol{P}}_{\Delta \bar{X}_{k}}\left(\delta V_{U}\right)+\hat{\boldsymbol{P}}_{\Delta \bar{X}_{k}}\left(\delta t_{r u}\right)}{\operatorname{tr}\left(\boldsymbol{G}_{I, E N U}\right)}
\end{aligned}
$$

Here, $\boldsymbol{R}_{\boldsymbol{k}}(\bullet)$ represents elements which correspond to pseudo-range and pseudo-range rate in measurement noise variance matrix; $\operatorname{tr}(\bullet)$ represents the sum of diagonal elements.

\section{Online Estimation of the System Noise Variance Matrix $\boldsymbol{Q}_{k}$}

Assume that the system noise $\boldsymbol{w}_{\boldsymbol{k}}$ is zero mean Gauss White noise, that is $\boldsymbol{w}_{\boldsymbol{k}} \sim N\left(0, \boldsymbol{Q}_{k}\right)$; Use the state prediction and the updated state estimation to update $\hat{\boldsymbol{Q}}_{k}$. Based on the maximum likelihood estimation (MMLE), we estimate the system noise covariance matrix $\hat{\boldsymbol{Q}}_{k}$. The estimation formula is as follows:

$$
\hat{\mathbf{Q}}_{k}=\frac{1}{N} \sum_{i=k-N+1}^{k}\left[\Delta \overline{\boldsymbol{X}}_{i} \Delta \overline{\boldsymbol{X}}_{i}^{T}+\boldsymbol{P}_{i}-\boldsymbol{\Phi}_{i, i-1} \boldsymbol{P}_{i-1} \boldsymbol{\Phi}_{i, i-1}^{T}\right]
$$

The formula (8) needs to store multiple historical data, which increases the storage space and leads to the real-time problem. Therefore, based on the modified version of this estimation proposed by Busse $^{[12]}$, the formula (8) will be modified to: 


$$
\hat{\boldsymbol{Q}}_{k}=\frac{1}{N} \sum_{j=0}^{N} \Delta \overline{\boldsymbol{X}}_{k-j} \Delta \overline{\boldsymbol{X}}_{k-j}^{T}-\boldsymbol{P}_{k}+\boldsymbol{\Phi}_{k, k-1} \boldsymbol{P}_{k-1} \boldsymbol{\Phi}_{k, k-1}^{T}=\hat{\boldsymbol{P}}_{\Delta \overline{\boldsymbol{X}}_{k}}+\boldsymbol{\Phi}_{k, k-1} \boldsymbol{P}_{k-1} \boldsymbol{\Phi}_{k, k-1}^{T}-\boldsymbol{P}_{k}
$$

Here, $\boldsymbol{\Phi}_{k, k-1}$ is state transition matrix; $\boldsymbol{P}_{k}$ is posteriori state estimation covariance matrix. When the $k$-th filtering is finished, through the GDOP ${ }_{I}$ and innovation according to section 3.1 in this paper, $\boldsymbol{R}_{k}$ is determined, then calculate $\boldsymbol{P}_{k}$ based on EKF; Estimate $\hat{\boldsymbol{Q}}_{k}$ according to the formula (9), it will be used for next filtering calculation.

\section{The experimental simulation and analysis}

To test the effectiveness of the algorithm, use the method described in section 3 to establish simulation platform: the observation point coordinates: $39.9631^{\circ}(N), 116.3047^{\circ}(E), 74.6725 \mathrm{~m}(H)$; the observation time: 1800s. The SiGe GN3S Sampler v3 receiver provides ephemeris data, original pseudo-range and doppler frequency shift. From the ephemeris data, we can calculate the satellite position, velocity and correct the influence of earth rotation. We can calculate pseudo-range rate by doppler frequency shift. In addition, the pseudo-range contains the ionospheric delay correction and the correction of satellite clock error. At the same observation point of SINS static base, the simulation condition for gyro random constant drift is $0.02^{\circ} / \mathrm{h}(1 \sigma)$, the noise of gyro is $0.02^{\circ} / \mathrm{h}(1 \sigma)$; the accelerometer random constant zero bias is $1 \times 10^{-4} \mathrm{~g}(1 \sigma)$, the noise of accelerometer is $1 \times 10^{-4} \mathrm{~g}(1 \sigma)$; through the simulation conditions, calculate position, velocity and attitude; the period of inertial navigation is $20 \mathrm{~ms}$. The parameters of EKF are set as follows: the period of filtering is 1s; the initial platform error angle is $0.5^{\circ}$; the initial position error is $10 \mathrm{~m}$; the initial velocity error are 0.1 $\mathrm{m} / \mathrm{s}, 0.1 \mathrm{~m} / \mathrm{s}$ and $0.5 \mathrm{~m} / \mathrm{s}$; the initial random noise variance of accelerometers and gyro are $(1 \% / \mathrm{h})^{2}$ and $\left(1 \times 10^{-2} \mathrm{~g}\right)^{2}$; the initial clock error and clock drift error of GPS are $30 \mathrm{~m}$ and $0.3 \mathrm{~m} / \mathrm{s}$; the initial variance of pseudo-range and pseudo-range rate are $(50 \mathrm{~m})^{2},(0.5 \mathrm{~m} / \mathrm{s})^{2}$. According to the section 1 , synchronize the SINS and GPS observation in SINS/GPS integrated navigation system. This paper will compare the proposed adaptive filtering method with the traditional EKF method: the method proposed in this paper uses the adaptive filtering to estimate $\boldsymbol{Q}_{k}$ and $\boldsymbol{R}_{\boldsymbol{k}}$ at the same time online; in the process of simulation, the total number of visible satellite is 4 , they are: $15-18-22-26$, the average GDOP value during the 1800s is 2.4587; the size of the IAE estimation window is $N=8$, the simulation results are shown in Fig.2.
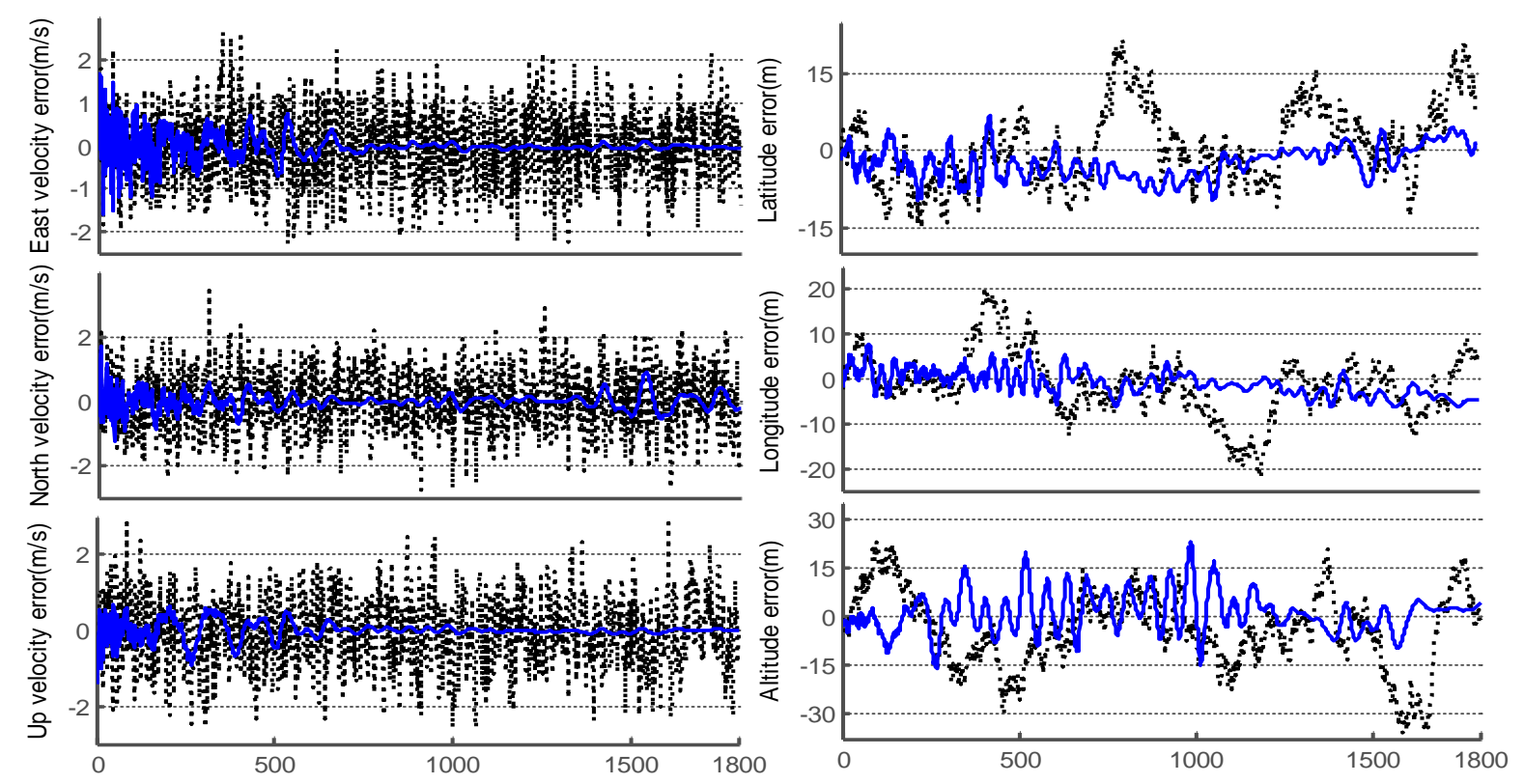

Fig.2 SINS / GPS tightly integrated navigation system error comparison

In Fig.2, the dotted line represents simulation curve of EKF method, we notice that the curve of velocity error vibrates seriously, the longitude and latitude error look slight, but the altitude error looks serious. The solid line represents simulation curves of AEKF method. Compared with EKF, we could conclude from the simulation results that: the precision of AEKF is better than EKF 
obviously .we could estimate $\boldsymbol{R}_{k}$ and $\boldsymbol{Q}_{k}$ through AEKF online, the position error and the velocity error all improve prominently, curves keep more smooth, errors decline overtly and the navigation effect keeps stable. In order to analyze different approaches quantificationally, calculate RMS of 800 1800 sample points, presented in Tab 1.

Tab 1: SINS / GPS tightly integrated navigation system error comparison (RMS)

\begin{tabular}{cccccccc}
\hline & \multicolumn{3}{c}{ Velocity error(m/s) } & & \multicolumn{3}{c}{ Position error(m) } \\
\cline { 2 - 4 } RMS & East & North & Up & & Latitude & Longitude & Altitude \\
\hline EKF & 0.7502 & 0.8626 & 0.8925 & & 8.1661 & 7.1593 & 12.4728 \\
\hline AEKF & 0.0790 & 0.2133 & 0.0654 & & 3.8626 & 3.2692 & 6.2265 \\
\hline
\end{tabular}

Compared with EKF method, the approach that estimates $\boldsymbol{R}_{k}$ and $\boldsymbol{Q}_{k}$ by adaption EKF presented in this paper could improve the stability of filter, velocity error, position error, and system precision.

\section{Conclusions}

Combined innovation information with the value of GDOP, we estimate measurement noise variance matrix $\boldsymbol{R}_{k}$ online; meanwhile, we revise system noise variance matrix $\boldsymbol{Q}_{k}$ using innovation information, thus realizing self-adaption EKF. As presented in simulation result, this method could achieve good effect that both velocity error and position error have been declined and the system accuracy has been improved apparently, at the same time, the stability of filter has been increased. In the next step, we will focus on the method of self-adaption EKF presented in this article in high dynamic condition. The method of self-adaption EKF could be used in fully integrated SINS/GPS navigation system as well.

\section{References}

[1] Xu T L. Adaptive Kalman Filter for INS/GPS Integrated Navigation System [J]. Applied Mechanics and Materials, 336(2013): 332-335.

[2] Shi E. An improved real-time adaptive Kalman filter for low-cost integrated GPS/INS navigation[C]. Measurement, Information and Control (MIC), International Conference on. IEEE, 2(2012): 1093-1098.

[3] Li Limin, Gong Wenbin, Liu Huijie, et al A Carrier Tracking Algorithm Based on Adaptive Extended Kalman Filter [J]. Acta Aeronautica Sinica, 33(7): 1319-1328, 2012.

[4] Yu M J. INS/GPS integration system using adaptive filter for estimating measurement noise variance [J]. Aerospace and Electronic Systems, IEEE Transactions on, 48(2): 1786-1792, 2012.

[5] Huo Guoping. On Investigation of GPS Positioning and Attitude Determination [D]. Beijing: Beijing Institute of Technology, 2013.

[6] Xie Gang. Principles of GPS and Receiver Design [M]. Beijing: Publishing House of Electronics Industry, 2009.

[7] Liu M, Xiong F. A fuzzy adaptive GPS/INS integrated navigation algorithm [J]. Procedia Engineering, 15(2011): 660-664.

[8] Gao S, Zhong Y, Li W. Robust adaptive filtering method for SINS/SAR integrated navigation system [J]. Aerospace Science and Technology, 15(6): 425-430, 2011.

[9] Yang Yuanxi. Adaptive Navigation and Kinematic Positioning [M]. Beijing: Surveying and Mapping Press, 2006.

[10] Zhao X, Qian Y, Zhang M, et al. An improved adaptive Kalman filtering algorithm for advanced robot navigation system based on GPS/INS[C]. Mechatronics Automation (ICMA), 2011 International Conference on. IEEE, (2011):1039-1044. 
[11]Zhou W, Qiao X, Meng F, et al. Study on SINS/GPS tightly integrated navigation based on Adaptive Extended Kalman Filter[C]. Information and Automation (ICIA), 2010 IEEE International Conference on. IEEE, (2010)2344-2347.

[12] Lu H, Zhan-rong J, Ming-ming W, et al. Adaptive extended Kalman filter based on genetic algorithm for tightly-coupled integrated inertial and GPS navigation[C]. Intelligent Computation Technology and Automation, 2009. ICICTA'09. Second International Conference on. IEEE, 1 (2009):520-524. 